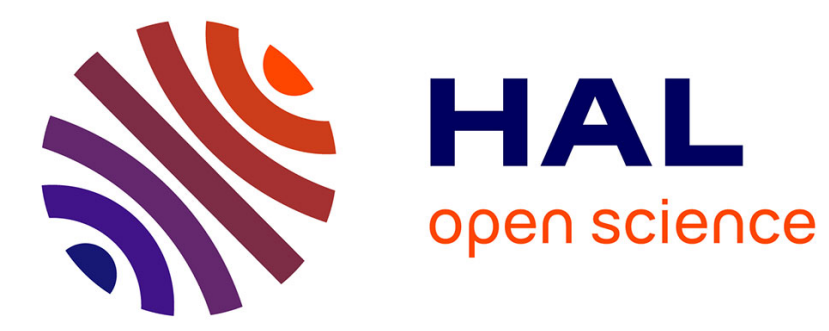

\title{
A Chiral Prussian Blue Analogue Pushes Magneto-Chiral Dichroism Limits
}

Matteo Atzori, Ivan Breslavetz, Kévin Paillot, Katsuya Inoue, Rikken G.L.J.A., Cyrille Train

\section{- To cite this version:}

Matteo Atzori, Ivan Breslavetz, Kévin Paillot, Katsuya Inoue, Rikken G.L.J.A., et al.. A Chiral Prussian Blue Analogue Pushes Magneto-Chiral Dichroism Limits. Journal of the American Chemical Society, 2019, 141 (51), pp.20022-20025. 10.1021/jacs.9b10970 . hal-03048317

\section{HAL Id: hal-03048317 https://hal.science/hal-03048317}

Submitted on 9 Dec 2020

HAL is a multi-disciplinary open access archive for the deposit and dissemination of scientific research documents, whether they are published or not. The documents may come from teaching and research institutions in France or abroad, or from public or private research centers.
L'archive ouverte pluridisciplinaire HAL, est destinée au dépôt et à la diffusion de documents scientifiques de niveau recherche, publiés ou non, émanant des établissements d'enseignement et de recherche français ou étrangers, des laboratoires publics ou privés. 


\title{
A Chiral Prussian Blue Analogue Pushes Magneto-Chiral Dichroism Lim- its
}

\author{
Matteo Atzori, ${ }^{\dagger, \star}$ Ivan Breslavetz, ${ }^{\dagger}$ Kévin Paillot, ${ }^{\dagger}$ Katsuya Inoue, ${ }^{\ddagger}$ Geert L. J. A. Rikken ${ }^{\dagger}$ and $C y-$ \\ rille $\operatorname{Train}^{\dagger, *}$ \\ $\dagger \quad$ Laboratoire National des Champs Magnétiques Intenses (LNCMI), Univ. Grenoble Alpes, INSA Toulouse, Univ. \\ Toulouse Paul Sabatier, EMFL, CNRS, Grenoble - Toulouse, France \\ ‡ Department of Chemistry and Center for Chiral Science, Hiroshima University, Hiroshima, Japan \\ Supporting Information Placeholder
}

\begin{abstract}
Here we report on the Magneto-Chiral Dichroism (MChD) detected with visible light on the chiral Prussian Blue Analogue $\left[\mathrm{Mn}^{\mathrm{II}}(X-p n \mathrm{H})\left(\mathrm{H}_{2} \mathrm{O}\right)\right]\left[\mathrm{Cr}^{\mathrm{III}}(\mathrm{CN})_{6}\right] \cdot \mathrm{H}_{2} \mathrm{O}(X=S$, $R ; p n=1,2$-propanediamine). Single crystals suitable for magneto-optical measurements were grown starting from enantiopure chiral ligands. X-ray diffraction and magnetic measurements confirmed the $2 \mathrm{D}$ structure of the material, its absolute configuration, and its ferrimagnetic ordered state below a critical temperature of $38 \mathrm{~K}$. Absorption and MChD spectra were measured between 450 and $900 \mathrm{~nm}$ from room temperature down to $4 \mathrm{~K}$. At $4 \mathrm{~K}$ the electronic spectrum features spin-allowed and spin-forbidden transitions of $\mathrm{Cr}^{\text {III }}$ centers, spin-forbidden transitions of the $\mathrm{Mn}^{\mathrm{II}}$ centers and metal-to-metal charge transfer bands. The MChD spectra below the magnetic ordering temperature exhibit intense absolute configuration-dependent $\mathrm{MChD}$ signals. The temperature dependence of these signals closely follows the material magnetization. Under a magnetic field of $0.46 \mathrm{~T}$, the most intense contribution to $\mathrm{MChD}$ represents $2.6 \% \mathrm{~T}^{-1}$ of the absorbed intensity, one of the highest values observed to date.
\end{abstract}

Investigating the interplay between chirality, light, and magnetism is a fascinating research challenge. All started in the $19^{\text {th }}$ century through the discoveries by Arago and Faraday of natural optical activity (NOA) and magneticallyinduced optical activity (MOA), respectively. ${ }^{1}$ NOA originates from a nonlocal optical response of a medium that lacks mirror symmetry, i.e. a chiral system, while MOA originates from the breaking of time-reversal symmetry by the magnetic field. ${ }^{2}$ This essential symmetry difference justifies that the direct link between the two phenomena was sought in vain by Louis Pasteur. ${ }^{1}$ However, these symmetry arguments allowed to predict the existence of a large class of nonreciprocal magneto-chiral effects, that can be observed in chiral systems possessing a magnetization, either spontaneous or induced by a magnetic field. ${ }^{2-4}$ Their optical manifestation features an absorption or emission of unpolarized light that depends on the relative orientation of the magnetic field $(\boldsymbol{B})$ with respect to the direction of light propagation $(\boldsymbol{k})$, and on the absolute configuration of the system. In absorption, this effect is called Magneto-Chiral Dichroism (MChD), ${ }^{2}$ while in refraction is called Magneto-Chiral Birefringence. ${ }^{3}$

MChD has been observed for frequencies ranging from microwaves ${ }^{5}$ to hard X-rays, ${ }^{6}$ underlining the universality of this effect. $^{2,5-13}$ To date, MChD in the Visible range was studied on a limited number of chiral molecular systems, namely nickel(II) sulfate hexahydrate, ${ }^{9}$ a terbium(III) complex, ${ }^{14} \mathrm{~J}$ aggregate of porphyrins, ${ }^{12}$ a $1 \mathrm{D} \mathrm{Cu}^{\mathrm{II}}-\mathrm{Cr}^{\mathrm{III}}$ coordination polymer, ${ }^{13}$ and $\left.\left[\mathrm{N}\left(\mathrm{CH}_{3}\right)\left(n-\mathrm{C}_{3} \mathrm{H}_{7}\right)_{2}\left(\mathrm{sec}-\mathrm{C}_{4} \mathrm{H}_{9}\right)\right]\left[\mathrm{Mn}^{\mathrm{II}} \mathrm{Cr}^{\mathrm{III}} \text { (oxalate) }\right)_{3}\right]$, a $2 \mathrm{D}$ ferromagnet with a Curie temperature of $6 \mathrm{~K}^{8}{ }^{8}$ Nonetheless, two major issues call for more in-depth studies of MChD in the UV-Visible-NIR region: i) the reverse effect, called magneto-chiral photochemistry, ${ }^{15}$ is considered as one of the possible mechanisms for the emergence of homochirality of life on Earth, ${ }^{1,12}$ ii) the use of MChD for optical readout of magnetic data has been envisioned but not yet demonstrated. ${ }^{16,17}$ For both issues, it is crucial to shed light on the origin of this effect and determine the microscopic parameters that govern $\mathrm{MChD}$ to design materials with strong MChD. For the later, it is essential to enhance the temperature where the spontaneous magnetization of the material drives the effect.

To tackle these challenges, we have investigated $\left[\mathrm{Mn}^{\mathrm{II}}(X\right.$ $\left.p n \mathrm{H})\left(\mathrm{H}_{2} \mathrm{O}\right)\right]\left[\mathrm{Cr}^{\mathrm{III}}(\mathrm{CN})_{6}\right] \cdot \mathrm{H}_{2} \mathrm{O}(X=S, R ; p n \mathrm{H}=1,2$-propanediamine $),{ }^{18,19}$ hereafter referred as $\mathbf{1}-(S)$ and $\mathbf{1}-(\boldsymbol{R})$. This material is a $2 \mathrm{D}$ chiral molecular ferrimagnet with a Curie temperature of $38 \mathrm{~K}^{19}$ The cyanide bridge ensures an efficient exchange interaction between metal ions and the chiral ligand triggers the absolute configuration of the material. ${ }^{10}$ In this bimetallic network, only $\mathrm{Mn}^{\mathrm{II}}$ is coordinated to a chiral ligand, thus providing nearest-neighbor chiral features to only one metal center. ${ }^{19}$

Single crystals of $\mathbf{1}$ are obtained by reacting hexacyanidochromate(III) with $\mathrm{Mn}^{\text {II }}$ and one of the two 1,2propanediamine enantiomers following published protocols. ${ }^{19}$ Slow liquid diffusion of the molecular components allows to obtain single-crystals with good optical transparency (Figure S1), a key feature for quantitative evaluation of MChD. The system crystallizes in the orthorhombic chiral space group $P_{2} 2_{1} 2_{1}$ with one $\left[\mathrm{Cr}^{\mathrm{III}}(\mathrm{CN})_{6}\right]^{3-}$ anion, one $\mathrm{Mn}^{\mathrm{II}}$ ion, one chiral $X-p n \mathrm{H}$ and one water molecule in the asymmetric unit (Figure 1a). In this structure, $\mathrm{Cr}^{\mathrm{III}}$ is surrounded by its six cyanide ligands while $\mathrm{Mn}^{\mathrm{II}}$ is coordinated to the monodentate chiral $X-p n H$ ligand in the apical posi- 
tion of a very slightly elongated octahedron (Figure a and S2). Accordingly, the immediate environment of both metal ions is not chiral, although $\mathrm{Mn}^{\mathrm{II}}$ is located closer to the stereogenic center than $\mathrm{Cr}^{\mathrm{III}}$. Repetition of the asymmetric unit leads to squared $2 \mathrm{D}$ layers (Figure $1 \mathrm{~b}$ and $\mathrm{S}_{3}$ ) that are at the origin of the long-range magnetic ordering.
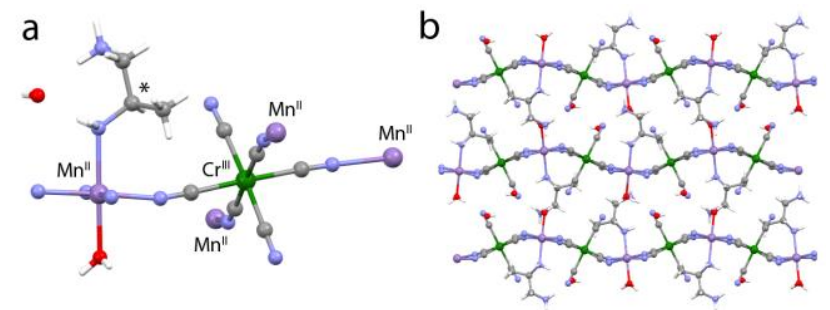

Figure 1. View of the structural moiety (a) and the $2 \mathrm{D}$ layered structure of $\mathbf{1}$ along the a crystallographic axis (b). Color codes: violet, $\mathrm{Mn}$; green, $\mathrm{Cr}$; blue, $\mathrm{N}$; red, $\mathrm{O}$; gray, $\mathrm{C}$; white, $\mathrm{H}$. The asterisk indicates the stereogenic center on the ligand.

Powder X-ray diffraction analysis on 1 confirmed the crystalline phase with experimental patterns in perfect agreement with the one calculated from single crystal XRD (Figures $\left.\mathrm{S}_{4}-\mathrm{S}_{5}\right) .{ }^{19}$ Magnetic measurements as a function of temperature and field confirmed the magnetic behavior of $1,{ }^{18}$ in particular the ferrimagnetic ordering below $38 \mathrm{~K}$ (Figure S6S8).

Single crystals of ca. $0.8 \times 0.8 \times 3 \mathrm{~mm}$ (Figure $\mathrm{S} 1$ ) were used to record absorption spectra in transmission mode along the c crystallographic axis as a function of the temperature (294-4.0 K) in the 450-900 $\mathrm{nm}$ spectral window to get insights on the electronic transitions of $\mathbf{1}$. The temperature variation of the absorption coefficient $\left(\mathrm{A}, \mathrm{cm}^{-1}\right)$ is reported in Figure 2a, while Figure $\mathrm{S} 9$ shows a comparison between the spectra at $294 \mathrm{~K}$ and $4.0 \mathrm{~K}$.

The electronic spectrum at room temperature shows two broad absorption peaks at $\lambda=490 \mathrm{~nm}$ and $608 \mathrm{~nm}$, the latter being shouldered, and a structured absorption spanning from $730 \mathrm{~nm}$ to $820 \mathrm{~nm}$ with two distinct peaks at $\lambda=782$ $\mathrm{nm}$ and $801 \mathrm{~nm}$. These features are similar to those reported in the seminal work of Yoshida and coworkers. ${ }^{19}$ The two broad peaks at high energy are attributed to the two low energy spin allowed $(\Delta S=0) d$ - $d$ transitions of octahedral $\mathrm{Cr}^{\text {III }}$ : $\lambda=490 \mathrm{~nm},{ }^{4} \mathrm{~T}_{1}\left({ }^{4} \mathrm{~F}\right) \leftarrow{ }^{4} \mathrm{~A}_{2}\left({ }^{4} \mathrm{~F}\right)$, and $\lambda=608 \mathrm{~nm},{ }^{4} \mathrm{~T}_{2}\left({ }^{4} \mathrm{~F}\right) \leftarrow$ ${ }^{4} \mathrm{~A}_{2}\left({ }^{4} \mathrm{~F}\right) .{ }^{20,21}$ According to previous reports on closely related compounds, the broad absorption peak at low energy can be attributed to a set of metal-to-metal charge transfer (MMCT) transitions. ${ }^{19,21-23}$
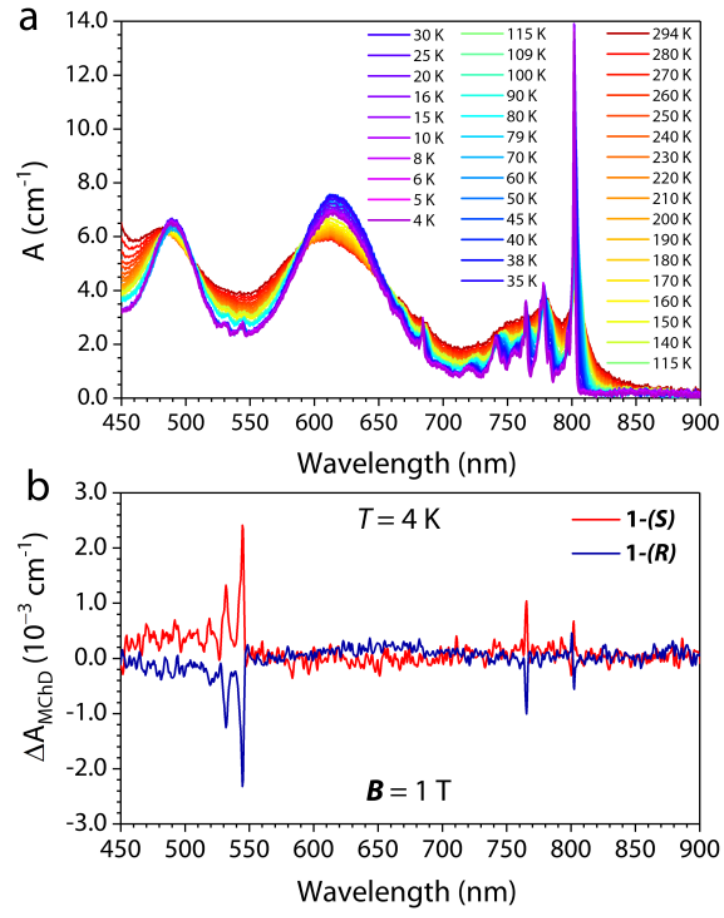

Figure 2. Temperature variation (see legend) of the absorption coefficient $A\left(\mathrm{~cm}^{-1}\right)$ for a single-crystal of $\mathbf{1}(\mathrm{a}) . \Delta \mathrm{A}_{\mathrm{MChD}}$ at 4.o K recorded on single crystals of $\mathbf{1}-(S)$ and $\mathbf{1}-(\boldsymbol{R})$ of different thickness (b).

By lowering the temperature, the shoulder of the $\lambda=608$ $\mathrm{nm}$ peak becomes a distinct peak at ca. $684 \mathrm{~nm}$ below $80 \mathrm{~K}$ (Figures 2a, S9, S10). Moreover, the MMCT band now appears as a series of narrow peaks. Finally, three peaks of weak intensities appear between the two spin-allowed $d$ - $d$ transitions of $\mathrm{Cr}^{\text {III }}$. The peak at $\lambda=684 \mathrm{~nm}$ corresponds to the $\mathrm{Cr}^{\text {III }}$ spinforbidden $(\Delta S= \pm 1){ }^{2} \mathrm{~T}_{2}\left({ }^{4} \mathrm{~F}\right) \leftarrow{ }^{4} \mathrm{~A}_{2}\left({ }^{2} \mathrm{G}\right)$ transition. Tanabe Sugano diagrams allow to analyze the spectroscopic features of the chromium centers. ${ }^{20}$ The energy of the lowest energy spin-allowed transition for $\mathrm{Cr}^{\mathrm{III}}, 615 \mathrm{~nm}$, provides the value of the crystal field parameter $\Delta_{O}, 16260 \mathrm{~cm}^{-1}$. The ratio between the two lowest energy spin-allowed transitions is 1.26(1), which corresponds to $\Delta_{O} / \mathrm{B}$ of $37 .{ }^{24}$ This allows to evaluate the position of the ${ }^{2} \mathrm{~T}_{2}\left({ }^{4} \mathrm{~F}\right) \leftarrow{ }^{4} \mathrm{~A}_{2}\left({ }^{2} \mathrm{G}\right)$ transition at ca. $690 \mathrm{~nm}$ $(\mathrm{E} / \mathrm{B}=33)$, which is in agreement with the experimental findings and validates the assignments. Finally, the three electronic transitions between $500 \mathrm{~nm}$ and $550 \mathrm{~nm}$ correspond to spin-forbidden transition of high-spin $\mathrm{Mn}^{\text {II }}$. $^{21,25}$ The other $\mathrm{Mn}^{\text {II }}$ expected transitions are hidden under the spin-allowed transitions of $\mathrm{Cr}^{\mathrm{III}}$ precluding any further fine analysis.

Magnetic circular dichroism measurements for $\mathbf{1}$ were already reported ${ }^{18}$ while natural circular dichroism spectra are reported and commented in the SI (Figure S11).

MChD measurements were performed on single crystals of $\mathbf{1}-(\boldsymbol{S})$ and $\mathbf{1}-(\boldsymbol{R})$ in light transmission configuration with a fixed light propagation vector $\boldsymbol{k}$ parallel to the $c$ crystallographic axis and an alternating magnetic field $\boldsymbol{B}(\Omega=1.5 \mathrm{~Hz})$ of amplitude $\pm 1.0 \mathrm{~T}^{26}$ Measurements were performed in the temperature range $4.0-165 \mathrm{~K}$. Figure $2 \mathrm{~b}$ shows a survey spectrum for the two enantiomers at $T=4.0 \mathrm{~K}$, featuring multiple MChD signals originating from the difference in the absorption of the electronic transitions at $\lambda=545,532$, and $520 \mathrm{~nm}$, 
as well as $\lambda=770$ and $802 \mathrm{~nm}$ (Figure S10). Measurements performed in the same conditions for $\mathbf{1}-(S)$ and $\mathbf{1}-(\boldsymbol{R})$ provides signals of opposite signs resulting in perfect mirror images.

Following the interpretation of the absorption spectra, it appears that the most intense MChD signal is associated to the $\mathrm{Mn}^{\mathrm{II}}$ absorber, the less intense well-defined low energy MChD signals to the MMCT bands, while the transitions associated to $\mathrm{Cr}^{\mathrm{III}}$ weakly contribute to the $\mathrm{MChD}$ signal. These findings give some clues about the mechanisms of MChD in the Visible. First, in accordance with previous measurements of $\mathrm{MChD}$ in this spectral range ${ }^{8}$ and in contrast with what was observed in the hard X-ray region, ${ }^{6}$ intense signals appear for metal ions with low orbital moments. Second, despite the elusive chirality of the metal centers themselves, the MChD signals are present and intense. This result assesses the possibility to exploit the non-local character of chirality to observe strong MChD signals. However, the decrease in intensity when comparing $\mathrm{Mn}$ and $\mathrm{Cr}$ signals, clearly indicates how MChD is sensitive to the distance between magnetic and chiral centers.

The temperature dependence of $\Delta \mathrm{A}_{\mathrm{MChD}}$ was investigated for the most intense signals $(510-560 \mathrm{~nm})$ for both $\mathbf{1}-(S)$ and $\mathbf{1}-(\boldsymbol{R})$ and the results are reported in Figure 3. Remarkably, the strongest signal at $\lambda=545 \mathrm{~nm}$ is clearly observed up to 42 $\mathrm{K}$ (Figure $3 \mathrm{~b}$ ), thus slightly above the ordering temperature of the material $\left(T_{c}=38 \mathrm{~K}\right)$.
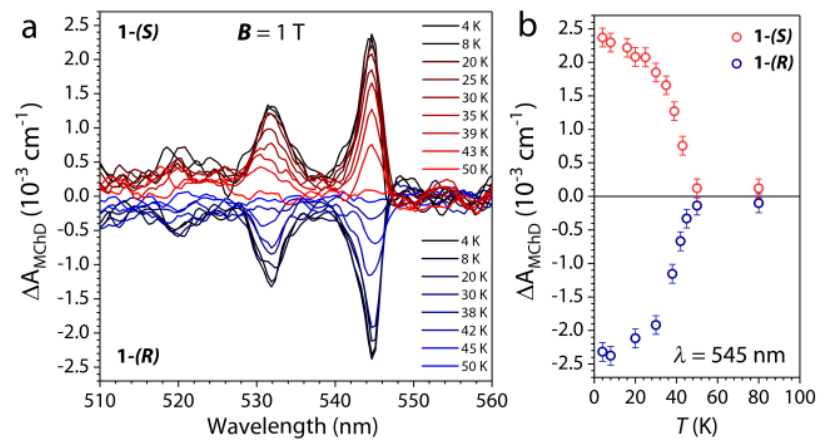

Figure 3. $\triangle \mathrm{A}_{\mathrm{MChD}}$ at different temperatures (see legend) for both $\mathbf{1}-(S)$ and $\mathbf{1}-(R)$ in the $\lambda=510-560 \mathrm{~nm}$ range (a). Temperature dependence of the $\Delta \mathrm{A}_{\mathrm{MChD}}$ signal at $\lambda=545 \mathrm{~nm}(\mathrm{~b})$.

To confirm that the enhancement of the MChD signal follows the spontaneous magnetization of the material, $\Delta \mathrm{A}_{\mathrm{MChD}}$ data were compared to magnetization data (Figure 4 ). Remarkably, the MChD signals are enhanced by a factor of 8 by lowering the temperature from 50 to $30 \mathrm{~K}$ (Figure 4a). An increase of the $\triangle \mathrm{A}_{\mathrm{MChD}}$ signal when entering into the magnetically ordered phase is due to the proportionality of $\triangle \mathrm{A}_{\mathrm{MChD}}$ to the cross-product $\boldsymbol{k} \cdot \boldsymbol{M}$, where $\boldsymbol{M}$ is the magnetization of the medium. $^{2}$ An enhancement of a factor of 17 was observed for the MChD signal of the compound $\left[\mathrm{Mn}^{\mathrm{II}} \mathrm{Cr}^{\mathrm{III}} \text { (oxalate) }{ }_{3}\right]^{-}$ when entering in to the ferromagnetic phase. ${ }^{8}$ However, considering the ferrimagnetic ordering nature of 1, which provides a low average magnetic moment (total ground spin state $S=1$ ) and the low anisotropic contribution to the magnetic moments involved, the enhancement is remarkable. Notably, $\left.\left[\mathrm{Mn}^{\mathrm{II}} \mathrm{Cr}^{\text {III }} \text { (oxalate) }\right]_{3}\right]^{-}$ordered ferromagnetically with a total ground spin state $S=4$.
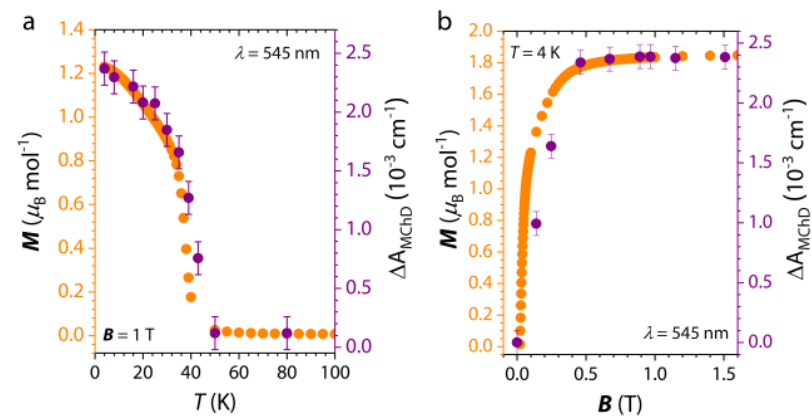

Figure 4. Temperature (a) and magnetic field (b) dependence of the $\Delta \mathrm{A}_{\mathrm{MChD}}$ signal $(\lambda=545 \mathrm{~nm})(\boldsymbol{B}=1.0 \mathrm{~T}, T=4 \mathrm{~K}$, respectively) for $\mathbf{1}-(S)$ compared to FC magnetization data.

To provide quantitative values of the MChD anisotropy factor $g_{M C h D},{ }^{9}$ which is defined as

$$
g_{M C h D}=\frac{(\mathrm{A}(\boldsymbol{B} \uparrow)-\mathrm{A}(\boldsymbol{B} \downarrow))}{1 / 2(\mathrm{~A}(\boldsymbol{B} \uparrow)+\mathrm{A}(\boldsymbol{B} \downarrow)) \boldsymbol{B}}=2 \frac{\Delta \mathrm{A}_{\mathrm{MChD}}}{\mathrm{A} \boldsymbol{B}}
$$

we have determined the effective absorption coefficients $A$ of the involved transitions by spectra deconvolution analysis (Figure S12). This allows quantification of $g_{M C h D}$ for each observed MChD signal according to eq. 1 without the spurious contribution of superimposed absorption bands that are not intrinsically involved (Table $\mathrm{S}_{1}$ ). Of particular relevance is the signal at $\lambda=545 \mathrm{~nm}$, that provides a value of $g_{M C h D}=0.012(1)$ $\mathrm{T}^{-1}(T=4 \mathrm{~K}, \boldsymbol{B}=1 \mathrm{~T})$, which is comparable to that recently observed through light emission for $\mathrm{a} \mathrm{Tb}^{\mathrm{III}}$ complex at $T=5 \mathrm{~K}$ under a much more intense magnetic field $\boldsymbol{B}=14 \mathrm{~T}$ (o.011 T $\left.{ }^{1}\right) .^{7}$ This is notable given the negligible orbital contribution to the magnetic moment of $\mathrm{Mn}^{\mathrm{II}}$ with respect to $\mathrm{Tb}^{\mathrm{III}}$. Considering that the magnetic field dependence of $\Delta \mathrm{A}_{\mathrm{MChD}}$ shows saturation already at $0.46 \mathrm{~T}$ (Figures $4 \mathrm{~b}$ and $\mathrm{S}_{13}$ ), the $g_{M C h D}$ for $\mathbf{1}$ at $\lambda=545 \mathrm{~nm}$ reaches a maximum value of $2.6 \% \mathrm{~T}^{-1}$ which represents a record for low applied fields.

In conclusion, we have investigated the magneto-optical properties of a $2 \mathrm{D}$-layered chiral molecular ferrimagnet through MChD spectroscopy. Several strong MChD signals are detected in the Visible range with opposite sign for the two enantiomers. These results seem to indicate that high orbital moments are not a key ingredient for the observation of MChD in this spectral region. Moreover, it appears that independent magnetic and chiral centers can lead to the observation of MChD, the vicinity of the two centers increasing the intensity of the signal. The intensity of the MChD signals follows the temperature and field dependence of the magnetization and remains strong up to its ordering temperature ( $38 \mathrm{~K}$ ) even for low applied fields (ca. o.5 T). Pushing the observation of MChD in an ordered magnet from $6 \mathrm{~K}$, as observed for the chiral magnet based on $\left[\mathrm{Mn}^{\mathrm{II}} \mathrm{Cr}^{\text {III }} \text { (oxalate) }{ }_{3}\right]^{7}$ 2D-layers, to $38 \mathrm{~K}$, as found here for $\mathbf{1}$, is an important step towards the use of $\mathrm{MChD}$ for the optical detection of magnetization using unpolarized light. These results can thus be considered a starting point for $\mathrm{MChD}$ investigations in rationally designed chiral molecular materials aimed at reaching high MChD anisotropy factor at temperatures viable for practical applications.

\section{ASSOCIATED CONTENT}

Supporting Information 
Additional Figures, Tables, Equations and Experimental Section as mentioned in the text. This material is available free of charge via the Internet at http://pubs.acs.org.

\section{AUTHOR INFORMATION}

\section{Corresponding Author}

matteo.atzori@lncmi.cnrs.fr

cyrille.train@lncmi.cnrs.fr

\section{Notes}

The authors declare no competing financial interests.

\section{ACKNOWLEDGMENT}

The French National Research Agency (ANR) is acknowledged for financial support through ChiraMolCo (ANR 15CE29-0006-02) and Monafer (ANR-18-CE09-0032) projects.

\section{REFERENCES}

(1) The Origin of Chirality in the Molecules of Life; Royal Society of Chemistry: Cambridge, 2008.

(2) Rikken, G. L. J. A.; Raupach, E. Observation of MagnetoChiral Dichroism. Nature 1997, 390, 493-494.

(3) Barron, L. D.; Vrbancich, J. Magneto-Chiral Birefringence and Dichroism. Mol. Phys. 1984, 51, 715-730.

(4) Pop, F.; Auban-Senzier, P.; Canadell, E.; Rikken, G. L. J. A.; Avarvari, N. Electrical Magnetochiral Anisotropy in a Bulk Chiral Molecular Conductor. Nat. Commun. 2014, 5, 3757.

(5) Tomita, S.; Sawada, K.; Porokhnyuk, A.; Ueda, T. Direct Observation of Magnetochiral Effects through a Single Metamolecule in Microwave Regions. Phys. Rev. Lett. 2014, 113, 235501.

(6) Sessoli, R.; Boulon, M.-E.; Caneschi, A.; Mannini, M.; Poggini, L.; Wilhelm, F.; Rogalev, A. Strong Magneto-Chiral Dichroism in a Paramagnetic Molecular Helix Observed by Hard X-Rays. Nat. Phys. 2015, 11, 69-74.

(7) Taniguchi, K.; Nishio, M.; Kishiue, S.; Huang, P.-J.; Kimura, S.; Miyasaka, H. Strong Magnetochiral Dichroism for Visible Light Emission in a Rationally Designed Paramagnetic Enantiopure Molecule. Phys. Rev. Mater. 2019, 3, 045202.

(8) Train, C.; Gheorghe, R.; Krstic, V.; Chamoreau, L.-M.; Ovanesyan, N. S.; Rikken, G. L. J. A.; Gruselle, M.; Verdaguer, M. Strong Magneto-Chiral Dichroism in Enantiopure Chiral Ferromagnets. Nat. Mater. 20o8, 7, 729734 .

(9) Rikken, G. L. J. A.; Raupach, E. Pure and Cascaded Magnetochiral Anisotropy in Optical Absorption. Phys. Rev. E 1998, 58, 5081-5084.
(10) Train, C.; Gruselle, M.; Verdaguer, M. The Fruitful Introduction of Chirality and Control of Absolute Configurations in Molecular Magnets. Chem. Soc. Rev. 2o11, 40, 3297.

Nakagawa, N.; Abe, N.; Toyoda, S.; Kimura, S.; Zaccaro, J.; Gautier-Luneau, I.; Luneau, D.; Kousaka, Y.; Sera, A.; Sera, M.; Inoue, K.; Akimitsu, J.; Tokunaga, Y.; Arima, T. Magneto-Chiral Dichroism of $\mathrm{CsCuCl}_{3}$. Phys. Rev. B 2017, 96, 121102.

Kitagawa, Y.; Segawa, H.; Ishii, K. Magneto-Chiral Dichroism of Organic Compounds. Angew. Chemie Int. Ed. 2011, 50, 9133-9136.

(13) Taniguchi, K.; Kishiue, S.; Kimura, S.; Miyasaka, H. LocalSite Dependency of Magneto-Chiral Dichroism in Enantiopure One-Dimensional Copper(II)-Chromium(III) Coordination Polymers. J. Phys. Soc. Japan 2019, 88, 093708.

(14) Wang, K.; Ma, F.; Qi, D.; Chen, X.; Chen, Y.; Chen, Y.-C.; Sun, H.-L.; Tong, M.-L.; Jiang, J. Chiral Bis(Phthalocyaninato) Terbium Double-Decker Compounds with Enhanced Single-Ion Magnetic Behavior. Inorg. Chem. Front. 2018, 5, 939-943.

(15) Rikken, G. L. J. A.; Raupach, E. Enantioselective Magnetochiral Photochemistry. Nature 20oo, 405, 932-935.

(16) Galán-Mascarós, J. R. Bring to Light. Nat. Phys. 2015, 11, 7-8.

(17) Barron, L. D. Chirality and Magnetism Shake Hands. Nat. Mater. 2008, 7, 691-692.

(18) Inoue, K.; Kikuchi, K.; Ohba, M.; Ōkawa, H. Structure and Magnetic Properties of a Chiral Two-Dimensional Ferrimagnet WithTC of $38 \mathrm{~K}$. Angew. Chemie Int. Ed. 2003, 42, 4810-4813.

(19) Yoshida, Y.; Inoue, K.; Kikuchi, K.; Kurmoo, M. Biomimetic Transformation by a Crystal of a Chiral Mn II -Cr III Ferrimagnetic Prussian Blue Analogue. Chem. Mater. 2016, 28, 7029-7038.

(20) Tanabe, Y.; Sugano, S. On the Absorption Spectra of Complex Ions II. J. Phys. Soc. Japan 1954, 9, 766-779.

(21) Lever, A. B. P.; Rice, S. A. Inorganic Electronic Spectroscopy. Phys. Today 1969, 22, 77-77.

(22) Robin, M. B.; Day, P. Mixed Valence Chemistry-A Survey and Classification. In Advances in Inorganic Chemistry and Radiochemistry; 1968; pp 247-422.

(23) Meng, Y.-S.; Sato, O.; Liu, T. Manipulating Metal-to-Metal Charge Transfer for Materials with Switchable Functionality. Angew. Chemie Int. Ed. 2018, 57, 12216-12226.

(24) Racah, G. Theory of Complex Spectra. II. Phys. Rev. 1942, 62, 438-462.

(25) Jørgensen, C. K. Absorption Spectrum of Manganese (II) Diethylenetriamine Complexes. Inorganica Chim. Acta 1969, 3, 313-314.

(26) Kopnov, G.; Rikken, G. L. J. A. A Multichannel MagnetoChiral Dichroism Spectrometer. Rev. Sci. Instrum. 2014, 85, 053106. 


\section{SYNOPSIS TOC}

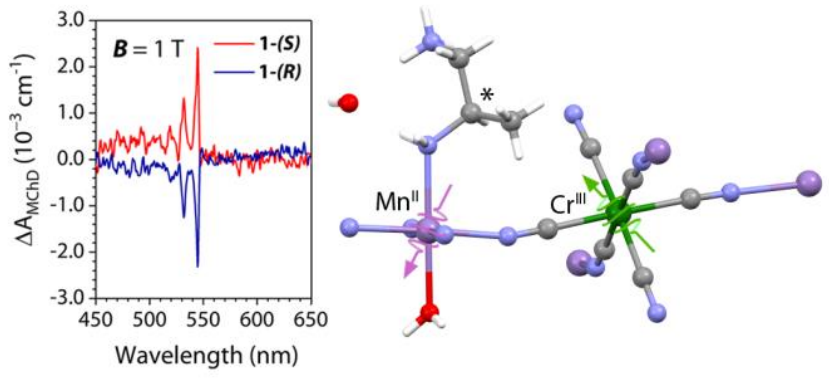

